\title{
An axiomatic approach to the measurement of envy
}

\author{
Kristof Bosmans $^{1}$ • Z. Emel Öztürk ${ }^{2}$
}

Received: 8 May 2014 / Accepted: 5 September 2017 / Published online: 21 September 2017

(C) The Author(s) 2017. This article is an open access publication

\begin{abstract}
We characterize a class of envy-as-inequity measures. There are three key axioms. Decomposability requires that overall envy is the sum of the envy within and between subgroups. The other two axioms deal with the two-individual setting and specify how the envy measure should react to simple changes in the individuals' commodity bundles. The characterized class measures how much one individual envies another individual by the relative utility difference (using the envious' utility function) between the bundle of the envied and the bundle of the envious, where the utility function that must be used to represent the ordinal preferences is the 'ray' utility function. The class measures overall envy by the sum of these (transformed) relative utility differences. We discuss our results in the light of previous contributions to envy measurement and multidimensional inequality measurement.
\end{abstract}

\section{Introduction}

An allocation is envy-free if no individual prefers another individual's commodity bundle to his own. The envy-freeness criterion was introduced to capture the ethical ideal of equality in a setting of ordinal preferences that are not comparable between

Kristof Bosmans

k.bosmans@maastrichtuniversity.nl

Z. Emel Öztürk

emel.ozturk@glasgow.ac.uk

1 Department of Economics, Maastricht University, Tongersestraat 53, 6211 LM Maastricht, The Netherlands

2 Adam Smith Business School, University of Glasgow, University Avenue, Glasgow G12 8QQ, UK 
individuals. ${ }^{1}$ Indeed, an envy-free allocation achieves equality in the sense that it leaves no individual worse off (in his own judgment) than any other individual. An alternative egalitarian interpretation, due to Kolm (1971), stresses that an envy-free allocation is such that it could have arisen by letting each individual choose a bundle from an identical opportunity set (e.g., the set of bundles in the given allocation).

Envy-freeness is a crude criterion. It distinguishes only two classes of allocations, those that are envy-free and those that are not. There are good reasons to consider envy measures that provide more discriminatory envy rankings of allocations. Since envyfreeness generalizes the idea of equality to the setting of ordinal and non-comparable preferences, the study of envy measures is a natural extension of the theory of inequality measurement. ${ }^{2}$ Further, allocations that are both envy-free and Pareto efficient are not guaranteed to exist in non-transferable-commodities or production settings. Hence, allocations in the Pareto efficient subset that minimize an envy measure constitute interesting compromises. ${ }^{3}$ Similarly, in the problem of allocating indivisible objects when money transfers are allowed, there is no allocation rule that is envy-free, strategyproof and budget-balanced. Again, an envy measure can be used to choose among the strategy-proof and budget-balanced allocation rules. ${ }^{4}$

We introduce a new class of envy measures. Throughout, we discuss the connections with envy measures proposed by Feldman and Kirman (1974), Chaudhuri (1986), Diamantaras and Thomson (1989), Tadenuma and Thomson (1995) and Fleurbaey (2008). Whereas these previous envy measures were proposed on the basis of their direct appeal, we use an axiomatic approach in order to make intuitions explicit. We develop our class in two steps.

First, we examine the consequences of imposing decomposability. This axiom requires that, for each partitioning of the population in two subgroups, envy in the total population can be written as the sum of the envy within subgroups and the envy between subgroups. In combination with a basic normalization axiom, decomposability implies that envy is measured by $\sum_{i} \sum_{j} E_{i j}$, where $E_{i j}$ measures how much

\footnotetext{
1 The seminal references are Tinbergen (1946), Foley (1967), Kolm (1971) and Varian (1974). See Arnsperger (1994) and Thomson (2011) for surveys. Note that the term 'envy' as we use it should be distinguished sharply from its everyday use, where the term designates the negative feeling aroused by another's good fortune. Indeed, an individual may well be envious in our sense without experiencing this negative feeling. Many authors have addressed this terminological issue. See, in particular, Varian (1974, pp. 66-67) and Kolm (1971, 1995).

2 Note that if there is only one commodity, say income, then the equal income distribution is the unique envy-free allocation. See Temkin (1986, 1993) and Ebert and Cowell (2004) for approaches to income inequality measurement that explicitly refer to envy-freeness.

3 Tadenuma (2002) studies this compromise solution in a formal setting similar to ours. In addition, he shows that the binary relation that naturally extends this solution (for any two allocations, prefer the Pareto dominant allocation if there is Pareto dominance, and prefer the envy-minimizing allocation if there is no Pareto dominance) violates the essential property of transitivity. Transitive binary relations that combine the Pareto principle and alternative equity principles have been proposed by, among others, Fleurbaey (2007) and Fleurbaey and Maniquet (2008, 2011).

4 Andersson et al. (2014) and Fujinaka and Wakayama (2015) propose allocation rules that are minimally manipulable among all envy-free and budget-balanced rules. We are not aware of studies that choose among the strategy-proof and budget-balanced rules by minimizing envy.
} 
individual $i$ envies individual $j$ and depends only on the bundles of $i$ and $j$ and the preferences of $i$. The value of $E_{i j}$ is zero if $i$ does not envy $j$, and positive otherwise.

Second, we formulate two axioms, betweenness and proportionality, that deal with envy comparisons in the simple two-individual setting. Assume that the two individuals are $i$ and $j$, and that $i$ envies $j$ but not vice versa. Betweenness demands that envy decreases if $i$ 's bundle improves or if $j$ 's bundle worsens according to $i$ 's preferences. Proportionality requires that, for cases where the bundles of individuals $i$ and $j$ are proportional to each other, envy is smaller if the radial distance between the bundles is smaller. We show that betweenness and proportionality are incompatible. We weaken the latter axiom to $r$-proportionality, which applies the idea of proportionality only if the bundles of $i$ and $j$ are proportional to a predetermined reference bundle $r$. Given anonymity, the axioms betweenness and $r$-proportionality imply that $E_{i j}$ is an increasing function of the ratio $u_{i}\left(x_{j}\right) / u_{i}\left(x_{i}\right)$, where $x_{i}$ and $x_{j}$ are the bundles of individuals $i$ and $j$ and $u_{i}$ is a 'ray' utility representation of $i$ 's ordinal preferences. The ray utility function depends on the chosen reference bundle $r$ and is singled out by the axioms as the utility representation to be used.

The next section introduces notation and provides an overview of the envy measures proposed in the literature. Sections 3 and 4 tackle the two steps discussed above. Section 5 combines the two steps into a single class of envy measures and discusses its properties. Section 6 concludes with a brief discussion of the connection with multidimensional inequality measurement.

\section{Preliminaries}

The set of individuals is $\mathcal{N}$, a finite subset of the set of positive integers. We assume that $\mathcal{N}$ has at least two members. Each individual $i$ in $\mathcal{N}$ has a commodity bundle $x_{i}$ in $X=\mathbb{R}_{++}^{m}$ and a preference relation $R_{i}$ on $X$. The expression $x R_{i} y$ means that bundle $x$ is at least as good as bundle $y$ according to individual $i$. The strict preference relation corresponding to $R_{i}$ is denoted by $P_{i}$ and the indifference relation by $I_{i}$. Individual $i$ is said to envy individual $j$ if $x_{j} P_{i} x_{i}$. The set $\mathcal{R}^{*}$ collects all complete, transitive, continuous and strictly monotonic preference relations. Each individual $i$ in $\mathcal{N}$ has a preference relation in $\mathcal{R}^{*}$. Additionally, we will make use of the flat preference relation $\bar{R}$, for which $x \bar{R} y$ for all bundles $x$ and $y$ in $X$. Because $\bar{R}$ is indifferent between all bundles, it never exhibits envy. Whenever we need to focus exclusively on the envy of a certain individual, we will replace the preference relation of every other individual by the flat preference relation $\bar{R}$. The set of all preference relations is $\mathcal{R}=\mathcal{R}^{*} \cup\{\bar{R}\}$.

For a set of individuals $N \subseteq \mathcal{N}$, we let $x_{N}=\left(x_{i}\right)_{i \in N}$ and $R_{N}=\left(R_{i}\right)_{i \in N}$. We refer to $\left(x_{N}, R_{N}\right)$ as a social state. We do not distinguish between social states that differ only with respect to the order in which the individuals are listed, e.g., $\left(x_{i}, x_{j}, R_{i}, R_{j}\right)$ and $\left(x_{j}, x_{i}, R_{j}, R_{i}\right)$ are treated as the same social state. The set $S$ collects all social states for each population that can be formed with the individuals in $\mathcal{N}$. That is, $S=\bigcup_{\emptyset \neq N \subseteq \mathcal{N}} X^{|N|} \times \mathcal{R}^{|N|}$.

We are interested in ranking the social states in $S$ on the basis of envy. To this end we study envy measures. An envy measure is a function $E: S \rightarrow \mathbb{R}$ that associates with each social state $s$ in $S$ a level of envy $E(s)$. 
We now explain how we use the flat preference $\bar{R}$ to single out the envy of one individual. Let $s=\left(x_{N}, R_{N}\right)$ be a social state in $S$. We consider two counterfactual social states that are induced by $s$. First, let $s_{i}$ denote the social state in which the preference relation of each individual $j \neq i$ is replaced by $\bar{R}$. That is, $s_{i}=\left(x_{1}, \ldots, x_{n}, \bar{R}, \ldots, \bar{R}, R_{i}, \bar{R}, \ldots, \bar{R}\right)$. Second, let $s_{i j}$ denote the social state for the two-individual population $\{i, j\} \subseteq N$ in which the preference relation of individual $j$ is replaced by $\bar{R}$. That is, $s_{i j}=\left(x_{i}, x_{j}, R_{i}, \bar{R}\right)$. In social state $s_{i}$, the only envy that occurs is that which individual $i$ experiences towards all other individuals in $N$. Likewise, in social state $s_{i j}$, the only envy that occurs is that which individual $i$ experiences towards individual $j$. We may therefore interpret $E\left(s_{i}\right)$ as the envy experienced by individual $i$ in social state $s$ and $E\left(s_{i j}\right)$ as the envy experienced by individual $i$ towards individual $j$ in social state $s$. We will refer to $E\left(s_{i}\right)$ as the individual envy of $i$ and to $E\left(s_{i j}\right)$ as the elementary envy that $i$ experiences towards $j$. Note that $E\left(s_{i}\right)$ and $E\left(s_{i j}\right)$ are well defined for each envy measure $E$ and each social state $s$ in $S$.

We define two basic axioms. More axioms will be introduced in the subsequent sections. Normalization requires the envy measure to attain the value of zero in envyfree social states and positive values in other social states. ${ }^{5}$

Normalization For each social state $\left(x_{N}, R_{N}\right)$ in $S$, we have $E\left(x_{N}, R_{N}\right) \geq 0$ with equality holding if and only if $x_{i} R_{i} x_{j}$ for all individuals $i$ and $j$ in $N$.

Anonymity demands that two social states featuring identical bundle-preference pairs exhibit the same level of envy. These two states may distribute these identical preference-bundle pairs differently over the same population or over altogether different populations (of the same size). For a bijection $\pi: N \rightarrow M$, and a social state $\left(x_{N}, R_{N}\right)$, we write $\pi\left(x_{N}\right)$ for $\left(x_{\pi(i)}\right)_{i \in N}$ and $\pi\left(R_{N}\right)$ for $\left(R_{\pi(i)}\right)_{i \in N}$.

Anonymity For each social state $\left(x_{N}, R_{N}\right)$ in $S$ and each bijection $\pi: N \rightarrow M$, we have $E\left(x_{N}, R_{N}\right)=E\left(\pi\left(x_{N}\right), \pi\left(R_{N}\right)\right)$.

To put the analysis of the next sections into perspective, we consider several envy measures that have been proposed in the literature. None of these measures has received axiomatic foundations. Each measure has been motivated instead by its immediate intuitive appeal. Table 1 presents the envy measures proposed by Feldman and Kirman (1974), Chaudhuri (1986) and Diamantaras and Thomson (1989). ${ }^{6}$ The table also shows how the measures fare with respect to our axioms.

All five measures rely on elementary envy as a basic building block. For the first four measures in Table 1, overall envy $E(s)$ equals the sum of all elementary envy values $\sum_{i \in N} \sum_{j \in N} E\left(s_{i j}\right)$. For the final measure in the table, overall envy $E(s)$ equals

\footnotetext{
5 In Sect. 5, we discuss an alternative normalization axiom, according to which the envy measure takes its minimum value only if all individuals are indifferent between all bundles.

6 Our formulation of the Diamantaras-Thomson measure follows Arnsperger (1994, Definition 5.4).
} 


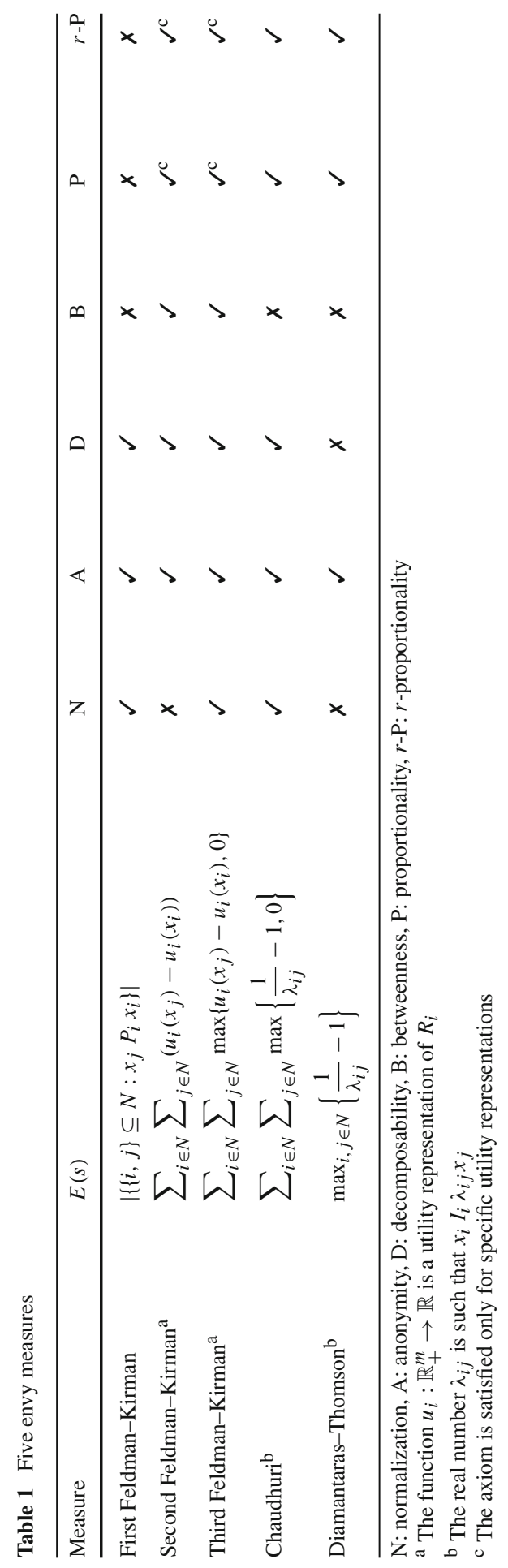


the maximum elementary envy $\max _{i, j \in N} E\left(s_{i j}\right) .{ }^{7}$ Let us focus now on how each of the measures defines elementary envy.

For the first Feldman-Kirman measure, which is a simple count of the instances of envy, the elementary envy $E\left(s_{i j}\right)$ equals 1 if individual $i$ envies individual $j$ and 0 if not. The measure clearly neglects the intensity of elementary envy, contrary to the next four measures in the table.

The second and third Feldman-Kirman measures require the existence of a cardinally significant utility representation of individual preferences. This stands in contrast to the other envy measures discussed in this section, as well as those developed in the next sections, which only require ordinal preference information. Let $u_{i}$ be the cardinal utility function of individual $i$. The elementary envy $E\left(s_{i j}\right)$ equals $u_{i}\left(x_{j}\right)-u_{i}\left(x_{i}\right)$ for the second Feldman-Kirman measure, and the same value truncated at zero for the third Feldman-Kirman measure. Hence, the former measure takes into account 'negative' elementary envy, i.e., the extent to which an individual prefers his own bundle to that of another, whereas the latter measure does not. ${ }^{8}$ A shortcoming of these two measures is the need for cardinal utility information.

The Chaudhuri and Diamantaras-Thomson measures focus on the factor $\lambda_{i j}$ by which the bundle of $j$ has to be shrunk in order for $i$ to stop envying $j$. For the Diamantaras-Thomson measure, elementary envy $E\left(s_{i j}\right)$ equals $\left(1 / \lambda_{i j}\right)-1$, where $\lambda_{i j}$ is such that $x_{i} I_{i} \lambda_{i j} x_{j}$. For the Chaudhuri measure, elementary envy is the same value truncated at zero. Again, the Diamantaras-Thomson measure takes into account 'negative' elementary envy, whereas the Chaudhuri measure does not. A shortcoming of these two measures is their arbitrary dependence on the particular procedure of shrinking the bundle of the envied. An a priori equally appealing procedure would be to focus on the factor by which the bundle of the envious has to be expanded in order for him to stop envying, but this procedure yields different results (see Sect. 4).

We proceed as follows. In Sect. 3 we characterize an envy measure that equates overall envy to the sum of all elementary envy values. The measure takes the form of the first four measures in Table 1. Measures that equate overall envy to the maximum elementary envy, as the Diamantaras-Thomson measure, or to the minimum elementary envy may be obtained as limiting cases (see Sect. 5). In Sect. 4 we consider axioms that impose properties on an envy measure only for the two-individual case. Using these axioms we characterize a measure of elementary envy that combines features of the utility difference approach of the second and third Feldman-Kirman measures

\footnotetext{
7 Hence, the five measures in Table 1 depend only indirectly on individual envy. The individual envy experienced by $i$ towards all other individuals in $N$ equals $E\left(s_{i}\right)=\sum_{j \in N} E\left(s_{i j}\right)$ for the first four measures in the table and $E\left(s_{i}\right)=\max _{j \in N} E\left(s_{i j}\right)$ for the final measure. In Sect. 5 we discuss measures by Tadenuma and Thomson (1995) and Fleurbaey (2008) that give a more substantial role to individual envy.

8 Feldman and Kirman (1974, p. 997) introduce their third measure with the explicit objective to not attribute positive ethical value to 'negative' elementary envy: "The [second] measure ... has the property that a man who isn't on the bottom rung of the economic ladder is compensated (in envy) by the misfortune of those below him. Such compensation may not seem entirely natural ...". As we will see in Sect. 3, jointly, the axioms normalization and decomposability fully eliminate the role of 'negative' elementary envy. In Sect. 5, we discuss an alternative approach that does give a role to 'negative' envy, but a negative rather than a positive role.
} 
and of the radial distance approach of the Chaudhuri and Diamantaras-Thomson measures. But at the same time the measure avoids the shortcomings of these approaches. Section 5 combines the results of the two preceding sections into a single class of envy measures and discusses its properties.

\section{Envy as the sum of elementary envy values}

Imagine a partitioning of the population into two subgroups, e.g., on the basis of region, ethnicity or sex. A standard question in the theory of inequality measurement applies here as well: how much of the overall envy in the population can be attributed to the envy within each of the two subgroups and how much to the envy between the two subgroups? The axiom decomposability requires that overall envy equals the sum of within-subgroup envy $W$ and between-subgroup envy $B .^{9}$

Decomposability For each social state $\left(x_{N}, R_{N}\right)$ in $S$ and each partition $\left\{N_{1}, N_{2}\right\}$ of $N$ with non-empty $N_{1}$ and $N_{2}$, we have

$$
E\left(x_{N}, R_{N}\right)=W+B
$$

where

$$
W=E\left(x_{N_{1}}, R_{N_{1}}\right)+E\left(x_{N_{2}}, R_{N_{2}}\right)
$$

and

$$
B=\sum_{i \in N_{1}} E\left(x_{i}, x_{N_{2}}, R_{i}, \bar{R}, \ldots, \bar{R}\right)+\sum_{i \in N_{2}} E\left(x_{i}, x_{N_{1}}, R_{i}, \bar{R}, \ldots, \bar{R}\right) .
$$

The within-subgroup term $W$ is itself the sum of the two subgroup envy values. The between-subgroup term $B$ measures the envy the members of one group experience towards the members of the other group and vice versa. Consider the first term in $B$. Because the preference relations of the members of $N_{2}$ are replaced by the flat preference relation $\bar{R}$, the value $E\left(x_{i}, x_{N_{2}}, R_{i}, \bar{R}, \ldots, \bar{R}\right)$ singles out the envy that individual $i$ experiences towards the members of $N_{2}$. The first term in $B$ sums these values over all $i$ in $N_{1}$. The term can therefore be interpreted as the envy experienced by the members of $N_{1}$ towards the members of $N_{2}$. The second term in $B$ similarly computes the envy experienced by the members of $N_{2}$ towards the members of $N_{1}$.

The following lemma says that if an envy measure satisfies normalization and decomposability, then it measures individual envy by the sum of the individual's elementary envy values.

\footnotetext{
9 For decomposability requirements in the theory of income inequality measurement, see, e.g., Bourguignon (1979), Cowell (1980) and Shorrocks (1980, 1984). The decomposability axiom used here most resembles that of Ebert (2010). While we, as is customary, state the axiom in terms of two subgroups, repeated application of the axiom allows a decomposition in any number of subgroups.
} 
Lemma 1 If E satisfies normalization and decomposability, then, for each social state $\left(x_{N}, R_{N}\right)$ in $S$ and each individual $i$ in $N$, we have

$$
E\left(s_{i}\right)=\sum_{j \in N} E\left(x_{i}, x_{j}, R_{i}, \bar{R}\right) .
$$

Proof The proof is by induction on the number of individuals.

Step 1 Let $N$ be such that $|N|=2$. Without loss of generality, let $N=\left\{i_{1}, i_{2}\right\}$. Let $s=\left(x_{i_{1}}, x_{i_{2}}, R_{i_{1}}, R_{i_{2}}\right)$ be a social state. Then,

$$
E\left(s_{i_{1}}\right)=E\left(x_{i_{1}}, x_{i_{2}}, R_{i_{1}}, \bar{R}\right)
$$

Step 2 Suppose the hypothesis holds for all $N$ such that $|N|=n$ with $n \geq 2$. We have to show that it holds for all social states with $n+1$ individuals.

Let $N^{\prime}$ be such that $\left|N^{\prime}\right|=n+1$ and let $s=\left(x_{N^{\prime}}, R_{N^{\prime}}\right)$ be a social state. Without loss of generality, let $N^{\prime}=\left\{i_{1}, i_{2}, \ldots, i_{n+1}\right\}$. Consider the partitioning of $N^{\prime}$ into $N_{1}^{\prime}=\left\{i_{1}, i_{2}, \ldots, i_{n}\right\}$ and $N_{2}^{\prime}=\left\{i_{n+1}\right\}$. By decomposability,

$$
\begin{aligned}
E\left(s_{i_{1}}\right)= & E\left(x_{N_{1}^{\prime}}, R_{i_{1}}, \bar{R}, \ldots, \bar{R}\right)+E\left(x_{i_{n+1}}, \bar{R}\right) \\
& +E\left(x_{i_{1}}, x_{i_{n+1}}, R_{i_{1}}, \bar{R}\right)+\sum_{k=2}^{n} E\left(x_{i_{k}}, x_{i_{n+1}}, \bar{R}, \bar{R}\right) \\
& +E\left(x_{i_{n+1}}, x_{N_{1}^{\prime}}, \bar{R}, \ldots, \bar{R}\right) .
\end{aligned}
$$

The second, fourth and final terms are equal to zero by normalization. Hence,

$$
E\left(s_{i_{1}}\right)=E\left(x_{N_{1}^{\prime}}, R_{i_{1}}, \bar{R}, \ldots, \bar{R}\right)+E\left(x_{i_{1}}, x_{i_{n+1}}, R_{i_{1}}, \bar{R}\right)
$$

By the induction hypothesis,

$$
E\left(x_{N_{1}^{\prime}}, R_{i_{1}}, \bar{R}, \ldots, \bar{R}\right)=\sum_{k=1}^{n} E\left(x_{i_{1}}, x_{i_{k}}, R_{i_{1}}, \bar{R}\right)
$$

Hence,

$$
E\left(s_{i_{1}}\right)=\sum_{k=1}^{n+1} E\left(x_{i_{1}}, x_{i_{k}}, R_{i_{1}}, \bar{R}\right) .
$$

The following proposition says that an envy measure satisfies normalization and decomposability if and only if overall envy equals the sum of all elementary envy values. 
Proposition 1 An envy measure E satisfies normalization and decomposability if and only if, for each social state $\left(x_{N}, R_{N}\right)$ in $S$, we have

$$
E\left(x_{N}, R_{N}\right)=\sum_{i \in N} \sum_{j \in N} E\left(x_{i}, x_{j}, R_{i}, \bar{R}\right),
$$

where $E\left(x_{i}, x_{j}, R_{i}, \bar{R}\right) \geq 0$ for all individuals $i$ and $j$ in $N$ with equality holding if and only if $x_{i} R_{i} x_{j}$.

Proof It is easy to see that the stated envy measure satisfies normalization and decomposability. We focus on the reverse implication. The proof is by induction on the number of individuals.

Step 1 Let $N$ be such that $|N|=2$. Without loss of generality, let $N=\left\{i_{1}, i_{2}\right\}$. Let $s=\left(x_{i_{1}}, x_{i_{2}}, R_{i_{1}}, R_{i_{2}}\right)$ be a social state. By decomposability,

$$
\begin{aligned}
E(s)= & E\left(x_{i_{1}}, R_{i_{1}}\right)+E\left(x_{i_{2}}, R_{i_{2}}\right) \\
& +E\left(x_{i_{1}}, x_{i_{2}}, R_{i_{1}}, \bar{R}\right)+E\left(x_{i_{2}}, x_{i_{1}}, R_{i_{2}}, \bar{R}\right) .
\end{aligned}
$$

The first and second terms are equal to zero by normalization. Hence,

$$
E(s)=E\left(x_{i_{1}}, x_{i_{2}}, R_{i_{1}}, \bar{R}\right)+E\left(x_{i_{2}}, x_{i_{1}}, R_{i_{2}}, \bar{R}\right) .
$$

Step 2 Suppose the hypothesis holds for all $N$ such that $|N|=n$ with $n \geq 2$. We have to show that it holds for all social states with $n+1$ individuals.

Let $N^{\prime}$ be such that $\left|N^{\prime}\right|=n+1$ and let $s=\left(x_{N^{\prime}}, R_{N^{\prime}}\right)$ be a social state. Without loss of generality, let $N^{\prime}=\left\{i_{1}, i_{2}, \ldots, i_{n+1}\right\}$. Consider the partitioning of $N^{\prime}$ into $N_{1}^{\prime}=\left\{i_{1}, i_{2}, \ldots, i_{n}\right\}$ and $N_{2}^{\prime}=\left\{i_{n+1}\right\}$. By decomposability,

$$
\begin{aligned}
E(s)= & E\left(x_{N_{1}^{\prime}}, R_{N_{1}^{\prime}}\right)+E\left(x_{i_{n+1}}, R_{i_{n+1}}\right) \\
& +\sum_{k=1}^{n} E\left(x_{i_{k}}, x_{i_{n+1}}, R_{i_{k}}, \bar{R}\right)+E\left(x_{i_{n+1}}, x_{N_{1}^{\prime}}, R_{i_{n+1}}, \bar{R}, \ldots, \bar{R}\right) .
\end{aligned}
$$

The second term is equal to zero by normalization. Using, in addition, the induction hypothesis,

$$
\begin{aligned}
E(s)= & \sum_{k=1}^{n} \sum_{\ell=1}^{n} E\left(x_{i_{k}}, x_{i_{\ell}}, R_{i_{k}}, \bar{R}\right) \\
& +\sum_{k=1}^{n} E\left(x_{i_{k}}, x_{i_{n+1}}, R_{i_{k}}, \bar{R}\right)+E\left(x_{i_{n+1}}, x_{N_{1}^{\prime}}, R_{i_{n+1}}, \bar{R}, \ldots, \bar{R}\right) .
\end{aligned}
$$


By Lemma 1,

$$
E\left(x_{i_{n+1}}, x_{N_{1}^{\prime}}, R_{i_{n+1}}, \bar{R}, \ldots, \bar{R}\right)=\sum_{k=1}^{n+1} E\left(x_{i_{n+1}}, x_{i_{k}}, R_{i_{n+1}}, \bar{R}\right)
$$

Hence,

$$
E(s)=\sum_{k=1}^{n+1} \sum_{\ell=1}^{n+1} E\left(x_{i_{k}}, x_{i_{\ell}}, R_{i_{k}}, \bar{R}\right)
$$

Proposition 1 says that overall envy equals the sum of all elementary envy values, but largely leaves open how to measure elementary envy. All it imposes in this respect is that 'negative' elementary envy, i.e., the extent to which an individual prefers his own bundle to that of another, is not taken into account. In the next section, we will consider axioms that give more substantial content to the concept of elementary envy.

\section{Measuring elementary envy}

Consider a setting with two individuals. We single out the envy that one individual experiences towards the other by replacing the latter's preference relation with the flat preference $\bar{R}$. We propose axioms that require the envy measure to react to simple changes in the bundles of the two individuals. The axioms bear on the envy measure $E$, but only directly impose properties on the elementary envy measure corresponding to $E$.

Betweenness requires the elementary envy that individual $i$ experiences towards individual $j$ to decrease if $i$ 's bundle improves or $j$ 's bundle worsens according to $i$ 's preferences. In terms of $i$ 's preferences, the new bundles lie 'in between' the original bundles. Figure 1 depicts an example where betweenness implies that $E\left(x_{i}, x_{j}, R_{i}, \bar{R}\right)>E\left(x_{i}^{\prime}, x_{j}^{\prime}, R_{i}, \bar{R}\right)$.

Betweenness For all individuals $i$ and $j$ in $\mathcal{N}$, all bundles $x_{i}, x_{j}, x_{i}^{\prime}$ and $x_{j}^{\prime}$ in $X$ and each preference relation $R_{i}$ in $\mathcal{R}$ such that $x_{j} P_{i} x_{i}$, we have that $x_{j} R_{i} x_{j}^{\prime}, x_{j}^{\prime} R_{i} x_{i}^{\prime}$ and $x_{i}^{\prime} R_{i} x_{i}$ imply $E\left(x_{i}, x_{j}, R_{i}, \bar{R}\right) \geq E\left(x_{i}^{\prime}, x_{j}^{\prime}, R_{i}, \bar{R}\right)$ with strict inequality holding whenever $x_{j} P_{i} x_{j}^{\prime}$ or $x_{i}^{\prime} P_{i} x_{i}$.

We emphasize an implication of betweenness. Let $u_{i}$ be an arbitrary utility representation of the preference relation $R_{i}$. Betweenness implies that the elementary envy $E\left(x_{i}, x_{j}, R_{i}, \bar{R}\right)$ experienced by individual $i$ towards $j$ can be expressed as a function only of the utility levels $u_{i}\left(x_{i}\right)$ and $u_{i}\left(x_{j}\right)$. That is, if $u_{i}\left(x_{i}\right)=u_{i}\left(x_{i}^{\prime}\right)$ and $u_{i}\left(x_{j}\right)=u_{i}\left(x_{j}^{\prime}\right)$ (as depicted in Fig. 2), then $E\left(x_{i}, x_{j}, R_{i}, \bar{R}\right)=E\left(x_{i}^{\prime}, x_{j}^{\prime}, R_{i}, \bar{R}\right)$. Note that the second and third Feldman-Kirman measures are in this functional form and satisfy betweenness. 
Fig. 1 Betweenness implies $E\left(x_{i}, x_{j}, R_{i}, \bar{R}\right)>$ $E\left(x_{i}^{\prime}, x_{j}^{\prime}, R_{i}, \bar{R}\right)$
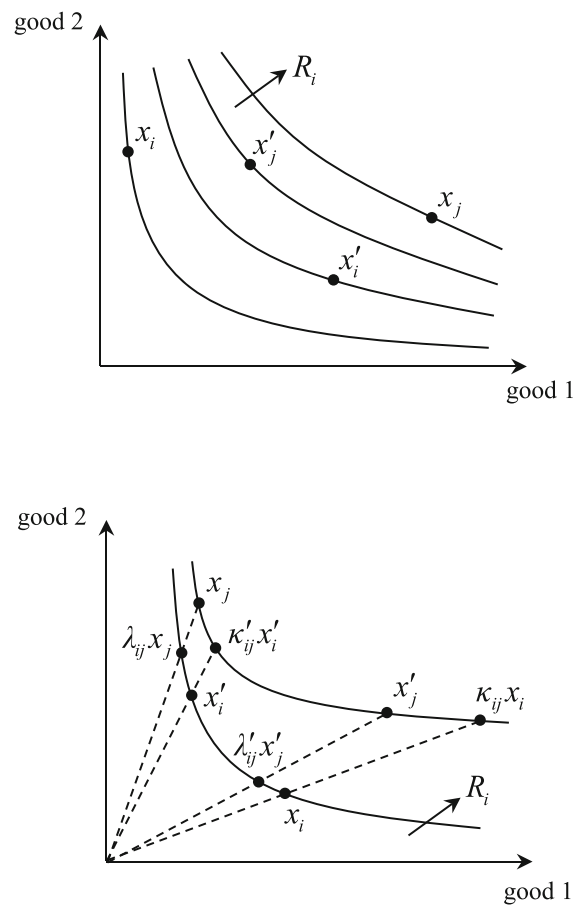

Fig. 2 Two clashing radial distance approaches

The next axiom captures the idea of gauging elementary envy by the radial distance between bundles. Consider two approaches. The first approach, as adopted in the Chaudhuri and Diamantaras-Thomson measures, measures the elementary envy experienced by $i$ towards $j$ using the factor $\lambda_{i j}$ by which $j$ 's bundle has to be shrunk in order for $i$ to stop envying $j$. That is, $\lambda_{i j}$ is such that $x_{i} I_{i} \lambda_{i j} x_{j}$, and the higher $\lambda_{i j}$, the lower the elementary envy of $i$. The second approach measures the elementary envy experienced by $i$ towards $j$ using the factor $\kappa_{i j}$ by which $i$ 's bundle has to be expanded in order for $i$ to stop envying $j$. That is, $\kappa_{i j}$ is such that $\kappa_{i j} x_{i} I_{i} x_{j}$, and the higher $\kappa_{i j}$, the higher the elementary envy of $i$. The two approaches are a priori equally appealing, but yield conflicting results. ${ }^{10}$ To see this, consider the social states $s=\left(x_{i}, x_{j}, R_{i}, \bar{R}\right)$ and $s^{\prime}=\left(x_{i}^{\prime}, x_{j}^{\prime}, R_{i}, \bar{R}\right)$ in Fig. 2. The first approach implies $E(s)<E\left(s^{\prime}\right)$ because $\lambda_{i j}>\lambda_{i j}^{\prime}$, whereas the second approach implies $E(s)>E\left(s^{\prime}\right)$ because $\kappa_{i j}>\kappa_{i j}^{\prime}{ }^{11}$

We do not make a choice among the two conflicting approaches. Instead, we formulate an axiom that is sufficiently weak to be consistent with both. The axiom only considers the cases where the bundles of the envied and envious are proportional to each other and says that a decrease of the radial distance between these two bundles

10 Tadenuma and Thomson (1995, p. 197) make a similar observation.

11 The two approaches do give the same result if the preference relation is homothetic. 
Fig. 3 Betweenness and proportionality clash

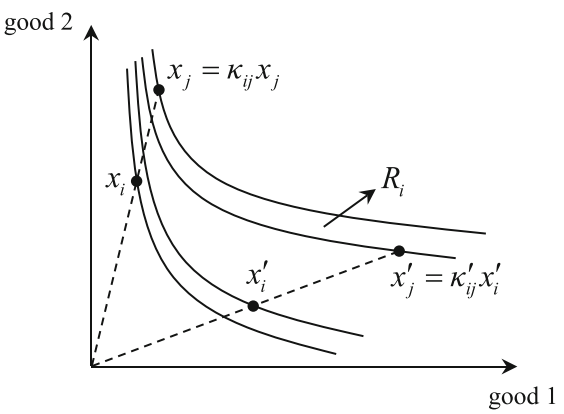

reduces elementary envy. In these cases $\lambda_{i j}=1 / \kappa_{i j}$ and the two above approaches coincide.

Proportionality For all individuals $i$ and $j$ in $\mathcal{N}$, all bundles $x_{i}, x_{j}, x_{i}^{\prime}$ and $x_{j}^{\prime}$ in $X$ such that $\kappa x_{i}=x_{j}$ and $\kappa^{\prime} x_{i}^{\prime}=x_{j}^{\prime}$ and all preference relations $R_{i}$ and $R_{i}^{\prime}$ in $\mathcal{R}$ such that $x_{j} P_{i} x_{i}$ and $x_{j}^{\prime} P_{i}^{\prime} x_{i}^{\prime}$, we have that $\kappa \geq \kappa^{\prime}$ implies $E\left(x_{i}, x_{j}, R_{i}, \bar{R}\right) \geq$ $E\left(x_{i}^{\prime}, x_{j}^{\prime}, R_{i}^{\prime}, \bar{R}\right)$ with strict inequality holding if and only if $\kappa>\kappa^{\prime}$.

However, betweenness and proportionality are incompatible: there is no envy measure that satisfies both axioms. Consider the social states $s=\left(x_{i}, x_{j}, R_{i}, \bar{R}\right)$ and $s^{\prime}=\left(x_{i}^{\prime}, x_{j}^{\prime}, R_{i}, \bar{R}\right)$ in Fig. 3. Betweenness implies $E(s)>E\left(s^{\prime}\right)$, whereas proportionality implies $E(s)<E\left(s^{\prime}\right)$ because $\kappa_{i j}<\kappa_{i j}^{\prime} \cdot{ }^{12}$

We treat betweenness as essential and therefore weaken proportionality. The following axiom requires all bundles to be proportional to a predetermined reference bundle $r$. Later we will argue that the axiom may be regarded as a minimum weakening of proportionality that is compatible with betweenness.

$r$-Proportionality There is a bundle $r$ in $X$ such that the following holds. For all individuals $i$ and $j$ in $\mathcal{N}$, all bundles $x_{i}, x_{j}, x_{i}^{\prime}$ and $x_{j}^{\prime}$ in $X$ proportional to $r$ and such that $\kappa x_{i}=x_{j}$ and $\kappa^{\prime} x_{i}^{\prime}=x_{j}^{\prime}$ and all preference relations $R_{i}$ and $R_{i}^{\prime}$ in $\mathcal{R}$ such that $x_{j} P_{i} x_{i}$ and $x_{j}^{\prime} P_{i}^{\prime} x_{i}^{\prime}$, we have that $\kappa \geq \kappa^{\prime}$ implies $E\left(x_{i}, x_{j}, R_{i}, \bar{R}\right) \geq E\left(x_{i}^{\prime}, x_{j}^{\prime}, R_{i}^{\prime}, \bar{R}\right)$ with strict inequality holding if and only if $\kappa>\kappa^{\prime}$.

Before proceeding, we define the 'ray' utility representation. Samuelson (1977), Pazner (1979), and Fleurbaey and Maniquet (2011), among others, have used this utility presentation in social welfare analysis with ordinal and non-comparable preferences. ${ }^{13}$ Let $\rho$ be a predetermined reference bundle in $X$. The ray utility level attained by individual $i$ with bundle $x_{i}$ and preference relation $R_{i}$ is equal to the real number $u_{\rho}\left(x_{i}, R_{i}\right)$ such that $i$ is indifferent between the fraction $u_{\rho}\left(x_{i}, R_{i}\right)$ of the bundle $\rho$ and his own bundle $x_{i}$. Figure 4 provides an illustration. Formally, for each preference

\footnotetext{
12 If the domain of preference relations is restricted to homothetic preferences relations, then the two axioms are compatible.

13 See, e.g., Malmquist (1953) and Kannai (1970) for earlier uses of the ray utility function.
} 
Fig. 4 The ray utility representation

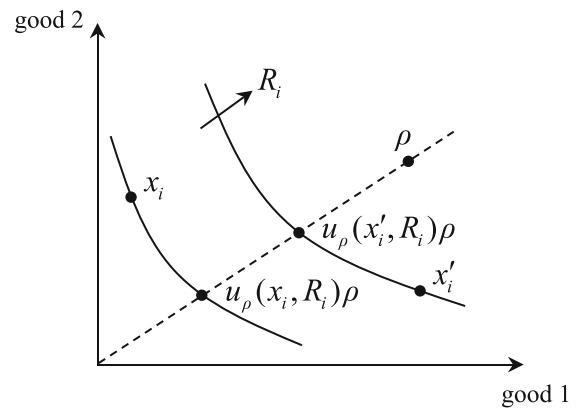

relation $R_{i}$ in $\mathcal{R} \backslash\{\bar{R}\}$ and each bundle $x_{i}$ in $X$, let $u_{\rho}\left(x_{i}, R_{i}\right)$ be the real number such that $x_{i} I_{i} u_{\rho}\left(x_{i}, R_{i}\right) \rho$. Let $u_{\rho}\left(x_{i}, \bar{R}\right)$ be equal to a positive constant for each bundle $x_{i}$ in $X$. Clearly, the function $u_{\rho}\left(\cdot, R_{i}\right)$ is a utility representation of the preference relation $R_{i}$.

The following proposition says that an envy measure satisfies betweenness and $r$ proportionality if and only if it measures the elementary envy experienced by individual $i$ towards $j$ by the ratio of $i$ 's ray utility levels associated with $j$ 's and $i$ 's bundles. Moreover, the reference bundle $\rho$ that determines the ray utility representation must be chosen such that $\rho=r$.

Proposition 2 Let $E$ be an envy measure that satisfies anonymity. Then E satisfies betweenness and $r$-proportionality if and only if there exists a strictly increasing function $f: \mathbb{R} \rightarrow \mathbb{R}$ such that, for all individuals $i$ and $j$ in $\mathcal{N}$, all bundles $x_{i}$ and $x_{j}$ in $X$ and each preference relation $R_{i}$ in $\mathcal{R}$ such that $x_{j} P_{i} x_{i}$, we have

$$
E\left(x_{i}, x_{j}, R_{i}, \bar{R}\right)=f\left(\frac{u_{r}\left(x_{j}, R_{i}\right)}{u_{r}\left(x_{i}, R_{i}\right)}\right) .
$$

Proof It is easy to see that the stated envy measure satisfies betweenness and $r$ proportionality. We focus on the reverse implication.

Let $r$ be a given bundle in $X$. Let $i$ and $j$ be individuals in $\mathcal{N}$, let $x_{i}, x_{i}^{\prime}, x_{j}$ and $x_{j}^{\prime}$ be bundles in $X$ and let $R_{i}$ and $R_{i}^{\prime}$ be preference relations in $\mathcal{R}$ such that $x_{j} P_{i} x_{i}$ and $x_{j}^{\prime} P_{i}^{\prime} x_{i}^{\prime}$. We have to show that

$$
E\left(x_{i}, x_{j}, R_{i}, \bar{R}\right) \geq E\left(x_{i}^{\prime}, x_{j}^{\prime}, R_{i}^{\prime}, \bar{R}\right)
$$

if and only if

$$
\frac{u_{r}\left(x_{j}, R_{i}\right)}{u_{r}\left(x_{i}, R_{i}\right)} \geq \frac{u_{r}\left(x_{j}^{\prime}, R_{i}^{\prime}\right)}{u_{r}\left(x_{i}^{\prime}, R_{i}^{\prime}\right)} .
$$

Then there exists a strictly increasing function $f$ as stated. Note that $f$ does not depend on $i$ and $j$ by anonymity. 
Let $y_{i}, y_{i}^{\prime}, y_{j}$ and $y_{j}^{\prime}$ be bundles in $X$ proportional to $r$ and such that $y_{i} I_{i} x_{i}, y_{i}^{\prime} I_{i}^{\prime} x_{i}^{\prime}$, $y_{j} I_{i} x_{j}$ and $y_{j}^{\prime} I_{i}^{\prime} x_{j}^{\prime}$. Such bundles exist since $R_{i}$ and $R_{i}^{\prime}$ are continuous and strictly monotonic. Let $\kappa$ and $\kappa^{\prime}$ be such that $\kappa y_{i}=y_{j}$ and $\kappa^{\prime} y_{i}^{\prime}=y_{j}^{\prime}$.

Suppose that Eq. (1) holds. We have to show that Eq. (2) holds as well. By betweenness, we have $E\left(x_{i}, x_{j}, R_{i}, \bar{R}\right)=E\left(y_{i}, y_{j}, R_{i}, \bar{R}\right)$ and $E\left(x_{i}^{\prime}, x_{j}^{\prime}, R_{i}^{\prime}, \bar{R}\right)=$ $E\left(y_{i}^{\prime}, y_{j}^{\prime}, R_{i}^{\prime}, \bar{R}\right)$. Hence, we obtain $E\left(y_{i}, y_{j}, R_{i}, \bar{R}\right) \geq E\left(y_{i}^{\prime}, y_{j}^{\prime}, R_{i}^{\prime}, \bar{R}\right)$. If $\kappa<\kappa^{\prime}$, then $E\left(y_{i}, y_{j}, R_{i}, \bar{R}\right)<E\left(y_{i}^{\prime}, y_{j}^{\prime}, R_{i}^{\prime}, \bar{R}\right)$ by $r$-proportionality. Hence, it must be that $\kappa \geq \kappa^{\prime}$. From the definition of $u_{r}$, it follows that $\kappa=u_{r}\left(y_{j}, R_{i}\right) / u_{r}\left(y_{i}, R_{i}\right)$ and $\kappa^{\prime}=u_{r}\left(y_{j}^{\prime}, R_{i}^{\prime}\right) / u_{r}\left(y_{i}^{\prime}, R_{i}^{\prime}\right)$. Since $u_{r}\left(x_{j}, R_{i}\right) / u_{r}\left(x_{i}, R_{i}\right)=u_{r}\left(y_{j}, R_{i}\right) / u_{r}\left(y_{i}, R_{i}\right)$ and $u_{r}\left(x_{j}^{\prime}, R_{i}^{\prime}\right) / u_{r}\left(x_{i}^{\prime}, R_{i}^{\prime}\right)=u_{r}\left(y_{j}^{\prime}, R_{i}^{\prime}\right) / u_{r}\left(y_{i}^{\prime}, R_{i}^{\prime}\right)$, we obtain Eq. (2).

Now, suppose that Eq. (2) holds. We have to show that Eq. (1) holds as well. Equation (2) implies that $\kappa=u_{r}\left(y_{j}, R_{i}\right) / u_{r}\left(y_{i}, R_{i}\right) \geq \kappa^{\prime}=u_{r}\left(y_{j}^{\prime}, R_{i}^{\prime}\right) / u_{r}\left(y_{i}^{\prime}, R_{i}^{\prime}\right)$. Since $\kappa \geq \kappa^{\prime}$, we have $E\left(y_{i}, y_{j}, R_{i}, \bar{R}\right) \geq E\left(y_{i}^{\prime}, y_{j}^{\prime}, R_{i}^{\prime}, \bar{R}\right)$ by $r$-proportionality. Using betweenness, we obtain Eq. (1).

The measure of elementary envy in Proposition 2 shares with the second and third Feldman-Kirman measures that it depends on the utility distance between the bundles of the envious and the envied. However, the utility representation used is not an arbitrary choice as in those measures. Rather, the radial distance idea inherent in the Chaudhuri and Diamantaras-Thomson measures singles out the ray utility representation.

Note that, for a given individual $i$, the criterion in Proposition 2 provides a complete ranking of all social states of the form $\left(x_{i}, x_{j}, R_{i}, \bar{R}\right)$. This means that any further strengthening of $r$-proportionality in the direction of proportionality will either lead to conflicts or is already implied by the combination of $r$-proportionality and betweenness. In other words, $r$-proportionality minimally weakens proportionality while ensuring compatibility with betweenness.

\section{Main result and discussion}

Our main result characterizes the class of envy measures satisfying normalization, anonymity, decomposability, betweenness and $r$-proportionality. The theorem is a straightforward combination of Propositions 1 and 2.

Theorem 1 An envy measure E satisfies normalization, anonymity, decomposability, betweenness and $r$-proportionality if and only if there exists a function $f: \mathbb{R}_{++} \rightarrow \mathbb{R}_{+}$ with $f$ strictly increasing on the interval $(1,+\infty)$ and $f(t)=0$ for each $t \leq 1$ such that, for each social state $\left(x_{N}, R_{N}\right)$ in $S$, we have

$$
E\left(x_{N}, R_{N}\right)=\sum_{i \in N} \sum_{j \in N} f\left(\frac{u_{r}\left(x_{j}, R_{i}\right)}{u_{r}\left(x_{i}, R_{i}\right)}\right) .
$$

To understand the role of the function $f$, it is useful to treat the ray utility ratio $u_{r}\left(x_{j}, R_{i}\right) / u_{r}\left(x_{i}, R_{i}\right)$ as a natural cardinalization of the measure of the elementary 
envy experienced by individual $i$ towards individual $j$. The more convex is $f$, the more sensitive is the envy measure $E$ to changes in larger elementary envy values (as measured by the ray utility ratio) relative to changes in smaller elementary envy values. Given a sufficiently convex $f$, the measure that equates overall envy to the largest elementary envy, as in the Diamantaras-Thomson measure, can be approximated arbitrarily closely. Similarly, choosing $f$ sufficiently concave delivers the other extreme that identifies overall envy with the minimum elementary envy.

We end this section by discussing three variants of Theorem 1. First, not all envy measures that have been proposed take the form of a sum over the elementary envy values. Fleurbaey's (2008, Chapter 2) measure equates the individual envy of $i$ to his maximum elementary envy $E\left(s_{i}\right)=\max _{j \in N} E\left(s_{i j}\right)$ and overall envy to the sum of all individual envy values $E(s)=\sum_{i \in N} E\left(s_{i}\right)$. Reversely, the envy measure underlying the equal-average-compensation solution of Tadenuma and Thomson (1995) equates the individual envy of $i$ to the sum of his elementary envy values $E\left(s_{i}\right)=\sum_{j \in N} E\left(s_{i j}\right)$ and overall envy to the maximum individual envy $E(s)=\max _{i \in N} E\left(s_{i}\right){ }^{14}$ These measures, contrary to the measures in Theorem 1, do not depend only on the elementary envy values, but also on their distribution over the individuals. Such a genuine role for the individual envy values can be allowed by replacing decomposability by two simple positive responsiveness axioms. The first axiom requires individual envy to increase if at least one individual's elementary envy increases, other things equal. The second axiom requires overall envy to increase if at least one individual envy increases, other things equal. These axioms lead to a general approach that allows different aggregations for the elementary envy values into individual envy and for the individual envy values into overall envy. We omit the straightforward formal treatment.

Second, we examine how the measure of elementary envy changes if we focus on the absolute distance between bundles instead of on the relative distance. The only change to the assumptions in Sect. 2 is that commodities can take negative or zero values in addition to positive values. We use $1_{m}$ to denote the $m$-vector with a one at each entry. Consider the following absolute version of $r$-proportionality. Let $x_{i}$ and $x_{i}^{\prime}$ be bundles such that $x_{i}=r+v 1_{m}$ and $x_{i}^{\prime}=r+v 1_{m}$, and let $x_{j}=x_{i}+\mu 1_{m}$ and $x_{j}^{\prime}=x_{i}^{\prime}+\mu^{\prime} 1_{m}$. Let $x_{j} P_{i} x_{i}$ and $x_{j}^{\prime} P_{i} x_{i}^{\prime}$. Then, according to the alternative axiom, $\mu \geq \mu^{\prime}$ implies $E\left(x_{i}, x_{j}, R_{i}, \bar{R}\right) \geq E\left(x_{i}^{\prime}, x_{j}^{\prime}, R_{i}^{\prime}, \bar{R}\right)$ with strict inequality holding if and only if $\mu>\mu^{\prime}$. Replacing $r$-proportionality by this alternative axiom in Theorem 1 yields the following class of measures: for each social state $\left(x_{N}, R_{N}\right)$ in $S$, we have

$$
E\left(x_{N}, R_{N}\right)=\sum_{i \in N} \sum_{j \in N} g\left(v_{r}\left(x_{j}, R_{i}\right)-v_{r}\left(x_{i}, R_{i}\right)\right),
$$

where $v_{r}\left(x_{i}, R_{i}\right)$ is the real number such that $x_{i} I_{i}\left(r+v_{r}\left(x_{i}, R_{i}\right) 1_{m}\right)$, and $g: \mathbb{R} \rightarrow \mathbb{R}$ is a function with $g$ strictly increasing on the interval $(0,+\infty)$ and $g(t)=0$ for each $t \leq 0$. Note that $v_{r}\left(\cdot, R_{i}\right)$ is a utility representation of the preference relation $R_{i}$.

\footnotetext{
14 Tadenuma and Thomson (1995) only compare envy-free social states. What we state here is the natural application of their proposal to social states that are not envy-free. To measure elementary envy, both Tadenuma and Thomson (1995) and Fleurbaey (2008) use approaches inspired by Chaudhuri (1986) and Diamantaras and Thomson (1989).
} 
The proof involves a simple adaptation of the proof of Proposition 2 and is therefore omitted.

Third, we discuss a variant of the envy measure that is increasing not only in elementary envy but also in 'negative' elementary envy, i.e., the extent to which an individual prefers his own bundle to that of another. ${ }^{15}$ We define an alternative normalization axiom that requires the envy measure to take its minimum value only if all individuals are indifferent between all bundles. ${ }^{16}$ Formally, for each social state $\left(x_{N}, R_{N}\right)$ in $S$, we have $E\left(x_{N}, R_{N}\right) \geq 0$ with equality holding if and only if $x_{i} I_{i} x_{j}$ for all individuals $i$ and $j$ in $N$. To ensure that overall envy increases with 'negative' envy, we define 'negative' versions of betweenness and $r$-proportionality. Negative betweenness says that, for all individuals $i$ and $j$ in $\mathcal{N}$, all bundles $x_{i}, x_{j}, x_{i}^{\prime}$ and $x_{j}^{\prime}$ in $X$ and each preference relation $R_{i}$ in $\mathcal{R}$ such that $x_{i} P_{i} x_{j}$, we have that $x_{i} R_{i} x_{i}^{\prime}, x_{i}^{\prime} R_{i} x_{j}^{\prime}$ and $x_{j}^{\prime} R_{i} x_{j}$ imply $E\left(x_{i}, x_{j}, R_{i}, \bar{R}\right) \geq E\left(x_{i}^{\prime}, x_{j}^{\prime}, R_{i}, \bar{R}\right)$ with strict inequality holding whenever $x_{i} P_{i} x_{i}^{\prime}$ or $x_{j}^{\prime} P_{i} x_{j}$. Negative $r$-proportionality says that, for all individuals $i$ and $j$ in $\mathcal{N}$, all bundles $x_{i}, x_{j}, x_{i}^{\prime}$ and $x_{j}^{\prime}$ in $X$ proportional to $r$ and such that $x_{i}=\kappa x_{j}$ and $x_{i}^{\prime}=\kappa^{\prime} x_{j}^{\prime}$ and all preference relations $R_{i}$ and $R_{i}^{\prime}$ in $\mathcal{R}$ such that $x_{i} P_{i} x_{j}$ and $x_{i}^{\prime} P_{i}^{\prime} x_{j}^{\prime}$, we have that $\kappa \geq \kappa^{\prime}$ implies $E\left(x_{i}, x_{j}, R_{i}, \bar{R}\right) \geq E\left(x_{i}^{\prime}, x_{j}^{\prime}, R_{i}^{\prime}, \bar{R}\right)$ with strict inequality holding if and only if $\kappa>\kappa^{\prime}$. If we replace in Theorem 1 normalization by its alternative and add negative betweenness and negative $r$-proportionality, then we obtain the following class of measures: for each social state $\left(x_{N}, R_{N}\right)$ in $S$, the envy measure $E$ takes the form in Eq. (3), where the function $f: \mathbb{R}_{++} \rightarrow \mathbb{R}_{+}$is strictly decreasing on the interval $(0,1)$, strictly increasing on the interval $(1,+\infty)$ and $f(1)=0$. Again, we omit the simple proof.

\section{Concluding remark}

We conclude with a remark on the connection between the measurement of envy and the so-called two-stage approach to the measurement of multidimensional inequality. ${ }^{17}$

The two-stage approach has so far only been applied to a setting with homogeneous preferences, ${ }^{18}$ but can in principle also be applied to a setting with heterogeneous preferences. The approach computes inequality in two stages: the first stage associates a utility level to the commodity bundle of each individual, and the second stage applies a uni-dimensional (income) inequality measure to the obtained vector of utilities. If preferences are heterogeneous, then envy measurement and the two-stage approach constitute manifestly distinct approaches to multidimensional inequality measurement. The former distinguishes between social states that are Pareto indifferent, i.e.,

\footnotetext{
15 The second Feldman-Kirman and Diamantaras-Thomson measures, by contrast, register the presence of 'negative' envy as a reduction of overall envy.

16 We are grateful to a referee for suggesting this axiom.

17 The two-stage approach was introduced by Maasoumi (1986). See Dardanoni (1995), Weymark (2006) and Bosmans et al. (2015) for discussions of the approach.

18 While contributions to the literature on multidimensional inequality measurement do not always explicitly define preferences, an interpretation in terms of (homogeneous) preferences is usually valid (see e.g., Tsui 1995, pp. 252-253).
} 
social states between which all individuals are indifferent, whereas the latter does not. ${ }^{19}$

However, if preferences are homogeneous, i.e., $R_{i}=R$ for each individual $i$, then the envy measures in Eqs. (3) and (4) produce particular two-stage inequality measures. The first stage computes the utility vectors, $\left(u_{r}\left(x_{i}, R_{i}\right)\right)_{i \in N}$ for (3) and $\left(v_{r}\left(x_{i}, R_{i}\right)\right)_{i \in N}$ for (4). For the second stage, it is easy to obtain well-known unidimensional inequality measures such as the absolute Gini index, the variance and the variance of logarithms as special cases of Eqs. (3) or (4). ${ }^{20}$

Acknowledgements We thank Jean-Jacques Herings, Erwin Ooghe and audiences in Maastricht (Maastricht University), New Delhi (11th Meeting of the Society for Social Choice and Welfare) and Barcelona (Universitat Autònoma de Barcelona) for useful comments.

Open Access This article is distributed under the terms of the Creative Commons Attribution 4.0 International License (http://creativecommons.org/licenses/by/4.0/), which permits unrestricted use, distribution, and reproduction in any medium, provided you give appropriate credit to the original author(s) and the source, provide a link to the Creative Commons license, and indicate if changes were made.

\section{References}

Andersson T, Ehlers L, Svensson L-G (2014) Budget balance, fairness, and minimal manipulability. Theor Econ 9:753-777

Arnsperger C (1994) Envy-freeness and distributive justice. J Econ Surv 8:155-186

Bosmans K, Decancq K, Ooghe E (2015) What do normative indices of multidimensional inequality really measure? J Publ Econ 130:94-104

Bourguignon F (1979) Decomposable income inequality measures. Econometrica 47:901-920

Chaudhuri A (1986) Some implications of an intensity measure of envy. Soc Choice Welf 3:255-270

Cowell FA (1980) On the structure of additive inequality measures. Rev Econ Stud 47:521-531

Dardanoni V (1995) On multidimensional inequality measurement. In: Dagum C, Lemmi A (eds) Income distribution, social welfare, inequality and poverty, research on economic inequality, vol 6. JAI Press, Stamford

Diamantaras D, Thomson W (1989) A refinement and extension of the no-envy concept. Econ Lett 30:103107

Ebert U (2010) The decomposition of inequality reconsidered: weakly decomposable measures. Math Soc Sci 60:94-103

Ebert U, Cowell FA (2004) Complaints and inequality. Soc Choice Welf 23:71-89

Feldman A, Kirman A (1974) Fairness and envy. Am Econ Rev 64:995-1005

Fleurbaey M (2007) Two criteria for social decisions. J Econ Theory 134:421-447

Fleurbaey M (2008) Fairness, responsibility and welfare. Oxford University Press, Oxford

Fleurbaey M, Maniquet F (2008) Fair social orderings. Econ Theory 34:25-45

Fleurbaey M, Maniquet F (2011) A theory of fairness and social welfare. Cambridge University Press, Cambridge

Foley D (1967) Resource allocation and the public sector. Yale Econ Essays 7:45-98

Fujinaka Y, Wakayama T (2015) Maximal manipulation of envy-free solutions in economies with indivisible goods and money. J Econ Theory 158:165-185

Kannai Y (1970) Continuity properties of the core of a market. Econometrica 38:791-815

Kolm S-C (1971) Justice et Equité. CEPREMAP, Paris

Kolm S-C (1995) The economics of social sentiments: the case of envy. Jpn Econ Rev 46:63-87

19 The tension between envy-freeness and Pareto indifference is well-known in the literature.

20 See Ebert (2010) for definitions of these and other measures covered by Eqs. (3) and (4). In a fixedpopulation setting, the envy measures and inequality measures correspond exactly. In a variable-population setting, division by $2|N|^{2}$ is required to obtain the inequality measures. 
Maasoumi E (1986) The measurement and decomposition of multi-dimensional inequality. Econometrica 54:991-998

Malmquist S (1953) Index numbers and indifference surfaces. Trabajos de Estadistica 4:209-242

Pazner EA (1979) Equity nonfeasible alternatives and social choice: a reconsideration of the concept of social welfare. In: Laffont J-J (ed) Aggregation and revelation of preferences. North-Holland, New York

Samuelson PA (1977) Reaffirming the existence of a "reasonable" Bergson-Samuelson social welfare functions. Economica 44:81-88

Shorrocks AF (1980) The class of additively decomposable inequality measures. Econometrica 48:613-625

Shorrocks AF (1984) Inequality decomposition by population subgroups. Econometrica 52:1369-1385

Tadenuma K (2002) Efficiency first or equity first? Two principles and rationality of social choice. J Econ Theory 104:462-472

Tadenuma K, Thomson W (1995) Refinements of the no-envy solution in economies with indivisible goods. Theory Decis 39:189-206

Temkin LS (1986) Inequality. Philos Publ Aff 15:99-121

Temkin LS (1993) Inequality. Oxford University Press, Oxford

Thomson W (2011) Fair allocation rules. In: Arrow KJ, Sen A, Suzumura K (eds) Handbook of social choice and welfare, vol 2. North-Holland, New York

Tinbergen J (1946) Redelijke Inkomensverdeling. De Gulden Pers, Haarlem

Tsui K-Y (1995) Multidimensional generalizations of the relative and absolute inequality indices: the Atkinson-Kolm-Sen approach. J Econ Theory 67:251-265

Varian HR (1974) Equity, envy, and efficiency. J Econ Theory 9:63-91

Weymark JA (2006) The normative approach to the measurement of multidimensional inequality. In: Farina F, Savaglio E (eds) Inequality and economic integration. Routledge, London 